\title{
Brainstem edema caused by traumatic carotid-cavernous fistula: A case report and review of the literature
}

\author{
JINLU YU $^{1}$, YUNBAO GUO ${ }^{1}$, SHUJIE ZHAO $^{2}$ and $\mathrm{KAN} \mathrm{XU}^{1}$ \\ ${ }^{1}$ Department of Neurosurgery; ${ }^{2}$ Intensive Care Unit, The First Affiliated Hospital of Jilin University, \\ Changchun, Jilin 130021, P.R. China
}

Received August 14, 2014; Accepted May 1, 2015

DOI: $10.3892 /$ etm.2015.2507

\begin{abstract}
Brainstem edema caused by traumatic carotid-cavernous fistula (TCCF) is rare, and there is little information available regarding its clinical characteristics. The present report describes the case of a 51-year-old man with TCCF, who presented with right exophthalmos and intracranial bruit for 1 week. One month prior to admission at hospital, he fractured the frontal and ethmoid sinuses. Digital subtraction angiography confirmed the diagnosis of TCCF, and magnetic resonance imaging (MRI) suggested edema on the right side of the pons. Five days after admission, the patient exhibited left hemiparesis, and MRI revealed aggravation of the brainstem edema. Following treatment with transarterial balloon embolization, the clinical symptoms, including hemiparesis, were relieved; at the 1-month follow-up, the brain edema had disappeared. The patient was normal at the 6-month follow-up. Following the report of the present case, we reviewed six additional cases previously reported in the literature and discussed the potential mechanisms of TCCF-associated brainstem edema. We conclude that occlusion of the superior petrosal sinus may contribute to brainstem edema caused by TCCF. Relief of the brainstem edema and brainstem edema-associated clinical symptoms can be achieved with transarterial coil or balloon embolization of the TCCF to reduce the drainage pressure in the brainstem veins.
\end{abstract}

\section{Introduction}

Traumatic carotid-cavernous fistula (TCCF) is a rare complication of head trauma that comprises an abnormal communication between the internal carotid artery and the cavernous sinus. It is characterized by the following cardinal symptoms: Exophthalmos, ocular pulsation, intracranial bruit, cranial nerve palsy, epistaxis, brain ischemia and intracranial hemorrhage (1);

Correspondence to: Dr Kan $\mathrm{Xu}$, Department of Neurosurgery, The First Affiliated Hospital of Jilin University, 71 Xinmin Avenue, Changchun, Jilin 130021, P.R. China

E-mail: jlyu@jlu.edu.cn

Key words: traumatic carotid-cavernous fistula, brainstem edema, hemiparesis however, the clinical presentation of brainstem edema in TCCF is rare, and only six cases to date have been reported (2-6). Clinical symptoms are primarily determined by the direction of the venous drainage. For instance, ophthalmic symptoms are more likely with venous drainage through the ophthalmic vein, and intracranial hemorrhage is more common with venous drainage via the cortical veins. In addition, high-flow shunting often leads to intracranial bruit (7). Due to its rarity, however, there is little information available regarding the clinical characteristics and the underlying mechanisms of brainstem edema caused by TCCF. The present report describes a rare case of TCCF with brainstem edema. In addition, we review the six known cases of brainstem edema in TCCF and discuss the potential mechanisms and prognosis of the condition.

\section{Case report}

A 51-year-old man presenting with right exophthalmos and intracranial bruit for 1 week was admitted to the First Affiliated Hospital of Jilin University (Changchun, China). The patient had a bone fracture in the frontal and ethmoid sinuses due to a car accident 1 month prior to admission. Cranial computed tomography (CT) bone window photography performed at the time of the accident revealed a bone fracture and hemorrhage in the frontal and ethmoid sinuses (Fig. 1). He received conservative treatment and was discharged following the alleviation of the symptoms.

On examination, the right eye was proptotic with conjunctival congestion, and the visual acuity in the right eye of the patient was 0.5 . Fundus examination revealed venous congestion and edema. A loud bruit in synchronization with the heartbeat was auscultated over the right orbit and the temporal bone. The eyeball was fixed with a dilated pupil that exhibited decreased pupillary light reflex. The cranial CT scan was normal (Fig. 2A). Fluid-attenuated inversion recovery magnetic resonance imaging (MRI) showed a hyperintense signal on the right side of the pons, consistent with brainstem edema (Fig. 2B). Digital subtraction angiography (DSA) showed that the cavernous sinus drained into the ophthalmic vein, inferior petrosal sinus and clival venous plexus. The enlarged vein in front of the brainstem drained to the straight sinus via the basal vein of Rosenthal and the vein of Galen. There was no filling of the superior petrosal sinus. Left carotid artery angiography was performed with cross compression of the right carotid artery, 


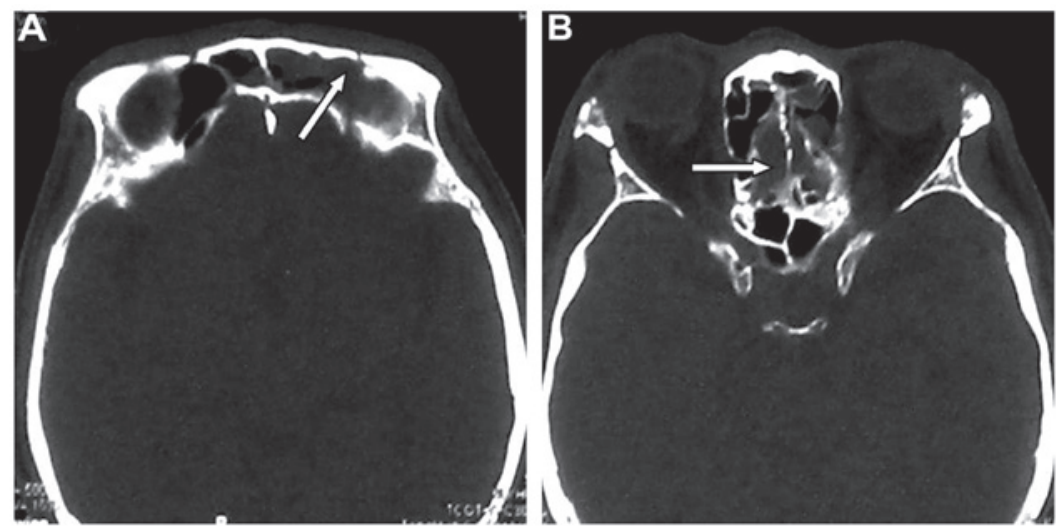

Figure 1. Images from cranial computed tomography bone window photography performed following the car accident, 1 month prior to hospital admission. Note the presence of bone fracture and hemorrhage (arrows) in the (A) frontal and (B) ethmoid sinuses.

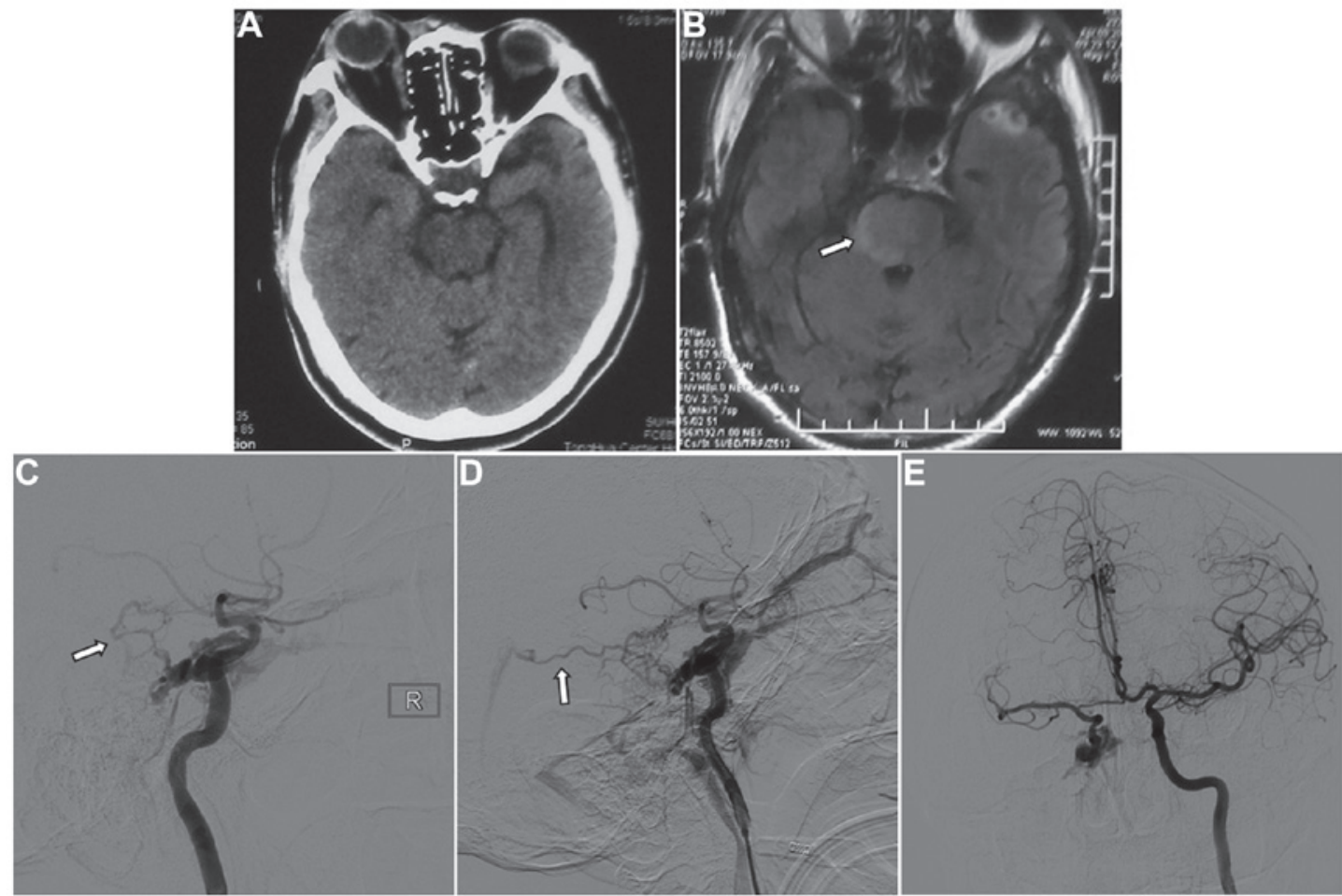

Figure 2. Images 1 week after the traumatic incident. (A) Computed tomography showed no abnormality. (B) Fluid-attenuated inversion recovery magnetic resonance imaging showed a hyperintense signal in the right side of the pons (arrow). (C and D) Digital subtraction angiography showed that the cavernous sinus drained into the ophthalmic vein, inferior petrosal sinus and clival venous plexus, and the basal vein of Rosenthal potentially enlarging it (arrows). There was no filling of the superior petrosal sinus. (E) Left carotid artery angiography with cross compression of the right carotid artery showed normal collateral circulation in the right carotid artery and no contrast delay in the middle cerebral artery.

demonstrating normal collateral circulation in the right carotid artery and no contrast delay in the middle cerebral artery (Fig. 2C-E). The patient was diagnosed with TCCF.

In order to improve the collateral circulation, the patient was treated with neck compression. Five days after admission, he developed left hemiparesis and right facial paralysis with shallow nasolabial folds. The muscle strength of the patient was $2 / 5$ in the extremities with positive pathological signs, and CT and MRI revealed aggravation of the brainstem edema (Fig. 3). The CCF was successfully embolized endovascularly with a detachable balloon on emergency. Angiography showed that the internal carotid artery was patent, and there was no contrast filling in the cavernous sinus (Fig. 4).
Following embolization, the ophthalmic signs were relieved, the intracranial bruit disappeared and the left hemiparesis gradually recovered. MRI performed 1 week postoperatively showed reduced brainstem edema (Fig. 5). At the 1-month follow-up, all symptoms, including hemiparesis, had disappeared, and MRI showed an absence of brainstem edema (Fig. 6). The patient was found to be normal at the 6-month follow-up performed via telephone.

\section{Discussion}

TCCF is not uncommon clinically, and the symptoms are mainly dependent on the direction of the cavernous sinus drainage. 

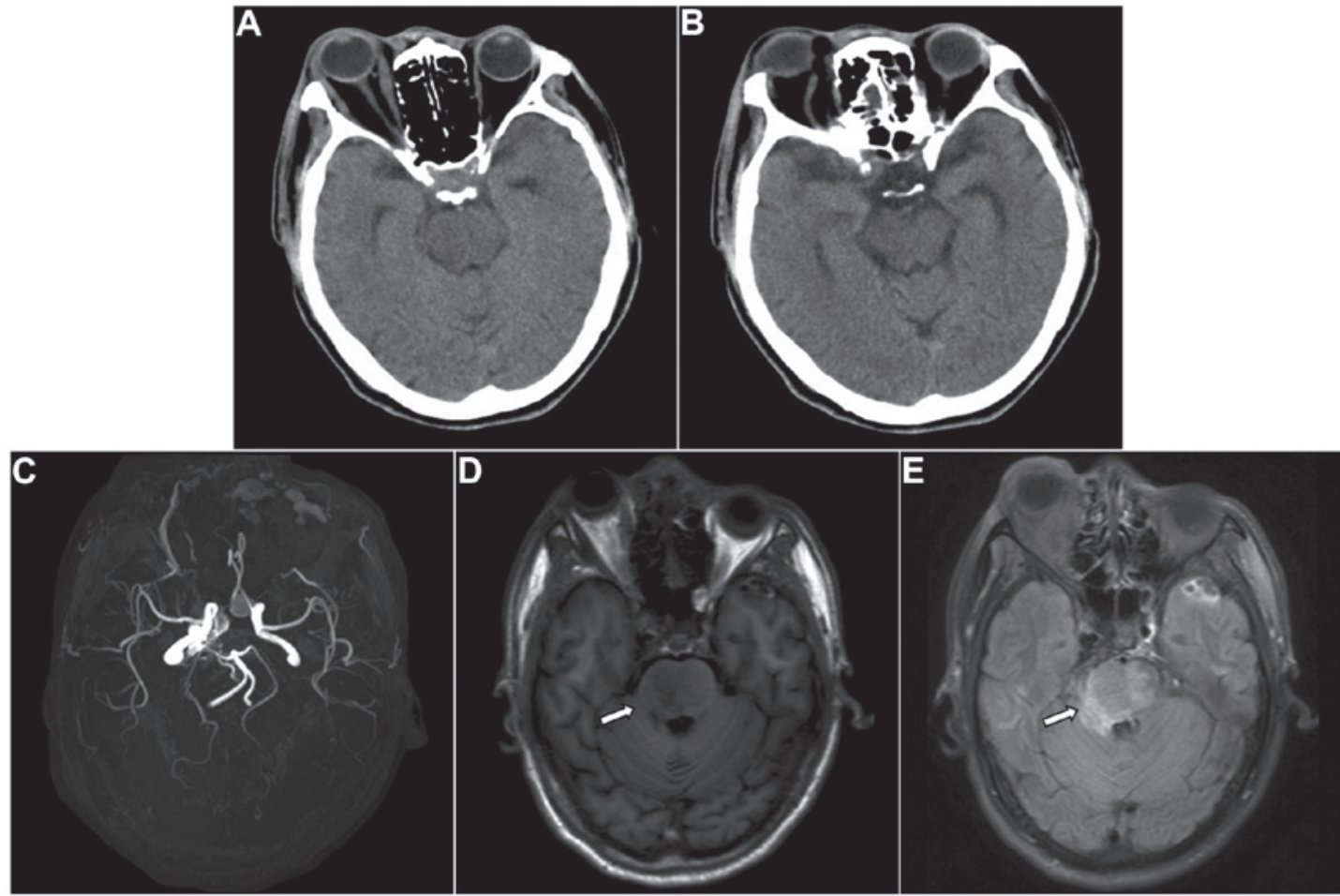

Figure 3. Radiological images 5 days after diagnosis of carotid-cavernous fistula, during which the patient had left hemiparesis. (A and B) Computed tomography showed no abnormality. (C) Magnetic resonance angiography revealed abnormal enlarged blood vessels in the cavernous sinus. (D) T1W1 and (E) fluid-attenuated inversion recovery magnetic resonance imaging showed aggravation of the brainstem edema (arrows).
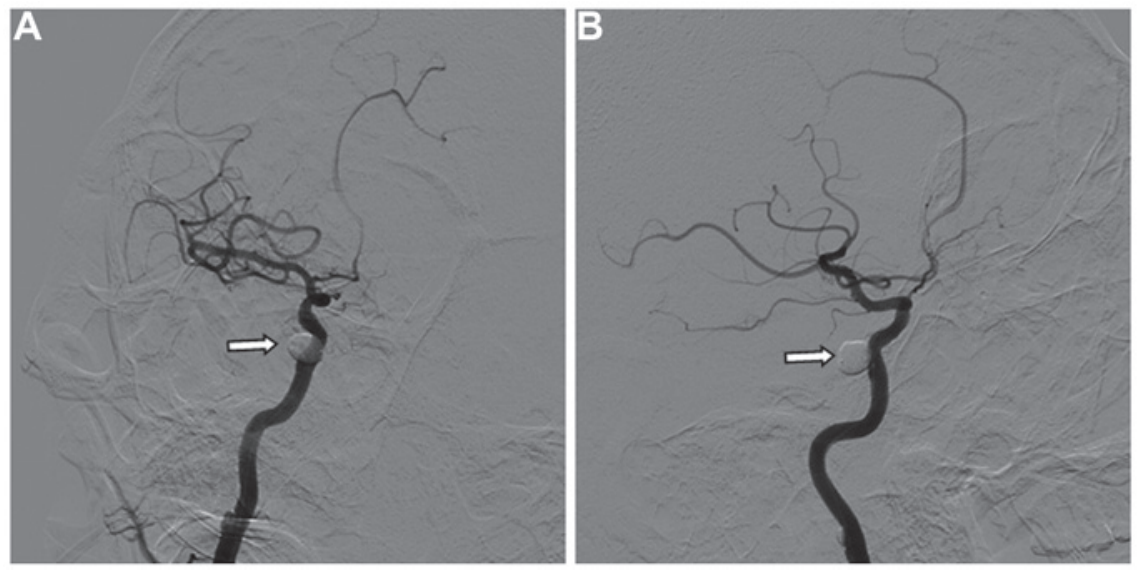

Figure 4. Angiography images during carotid-cavernous fistula embolization showed satisfactory results. (A and B) Oblique images revealed that the internal carotid artery was patent, and the fistula was occluded.

The cavernous sinus lies laterally to the pituitary gland and sphenoid bone and extends from the superior orbital fissure to the tip of the petrous bone. The cavernous sinus receives blood from the superior and inferior ophthalmic veins, superficial cortical vein and sphenoparietal sinus and drains into the superior and inferior petrosal sinus and pterygoid venous plexus. The $\mathrm{C} 4$ segment of the internal carotid artery passes through the cavernous sinuses, and they communicate with each other via the intercavernous sinus (8). When a sphenoid bone fracture punctures the internal carotid artery or a pseudoaneurysm is ruptured, arterial blood can enter the cavernous sinus, causing a TCCF. As high-flow and high-pressure arterial blood enters the cavernous sinus, an intracranial bruit can occur at the fistula. In addition, the hemodynamic changes in the cavernous sinus can reverse flow into tributaries. Reflux into the ophthalmic vein can lead to ophthalmic signs, and reflux into the ulnar vein can cause enlargement of the vein on the surface of the brain and cerebral hemorrhage (9). Due to these anatomic and hemodynamic characteristics of TCCF, patients with TCCF often exhibit atypical clinical symptoms as a result of the intracranial hemorrhage, in addition to typical clinical symptoms such as exophthalmos and intracranial bruit (10-12); however, the clinical symptoms of TCCF patients with brainstem edema are rarely reported, and the mechanisms and prognosis of TCCF with brainstem edema remain unclear. Here, we review the clinical characteristics of 

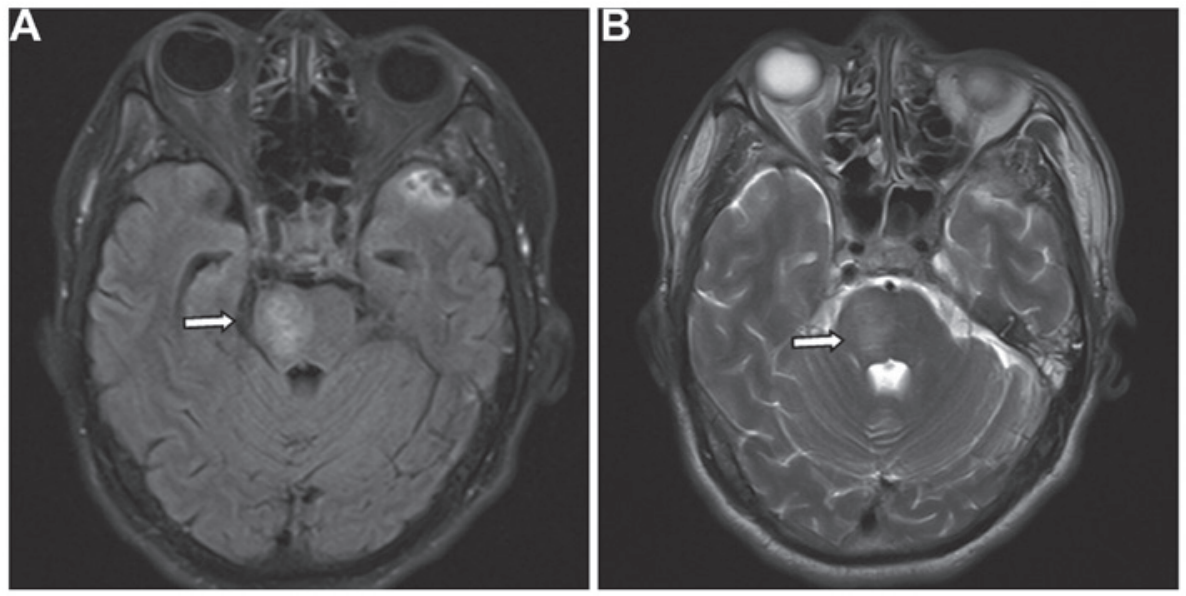

Figure 5. (A) Fluid-attenuated inversion recovery and (B) T2WI magnetic resonance imaging performed 1 week after embolization revealed reduced brainstem edema (arrows).
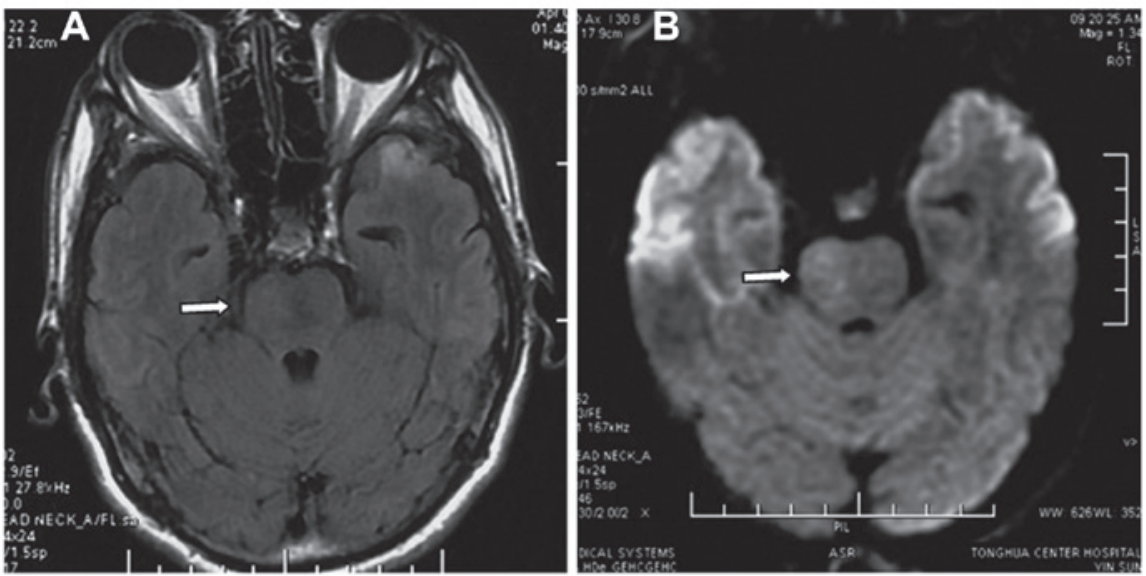

Figure 6. (A) Fluid-attenuated inversion recovery and (B) diffusion-weighted magnetic resonance imaging performed 1 month after embolization showed an absence of brainstem edema (arrows).

the present case along with the other six cases available from the literature (2-6).

TCCF-associated brainstem edema appears as a diffuse lesion with unclear boundary and exhibits venous congestive infarction on radiological images. This is similar to that caused by intracranial sinus thrombosis and venous thrombosis (13). Due to its rarity, elucidation of the mechanisms underlying brainstem edema caused by TCCF has been difficult. TCCF is not the only cause of brainstem edema, as cavernous sinus dural arteriovenous fistula (CSDAVF) has also been reported to cause brainstem edema $(14,15)$. In a retrospective study of 54 patients with CSDAVF, Miyamoto et al (15) reported that the main manifestation of venous congestive infarction was brainstem edema, which was primarily caused by insufficient venous collaterals, venous thrombosis, varicose, basilar venous hypoplasia and increased venous flow; however, brainstem edema following TCCF is not completely due to increased venous flow in the brainstem, as venous congestion, although rare, can occur despite an increase in venous flow in the superior and inferior petrosal sinus following the entry of arterial blood into the cavernous sinus (5). Increased blood flow in the periphery of the brainstem is therefore not the major cause of brainstem edema following TCCF.
In all six previous cases with brainstem edema caused by TCCF (Table I), thrombosis in the superior petrosal sinus occurred. Although thrombosis in the petrosal vein most commonly occurs in CSDAVF, thrombosis in the superior petrosal sinus was also found in CSDAVF (15). Consistent with the literature, the present case exhibited occlusion of the superior petrosal sinus on DSA. When the superior petrosal sinus is occluded, high-flow arterial blood mainly drains to the superior and inferior ophthalmic veins, inferior petrosal sinus and pterygoid plexus, thereby causing severe ophthalmic signs. The tributaries of the superior petrosal sinus drain to the brainstem veins; when they are occluded, the collaterals in the brainstem need to be re-established via the venous plexus in the periphery of the brainstem (16). In addition, small veins that communicate with the cavernous sinus are enlarged and drain the cavernous sinus. In the present case, the veins in front of the brainstem were enlarged and drained to the basal vein of Rosenthal. Ract et al (6) reported that the edema in the brainstem and basal ganglion was induced by drainage via the ulnar vein. Furthermore, following occlusion of the superior petrosal sinus, drainage to the inferior petrosal sinus increases, thus leading to impaired venous drainage in the periphery of the brainstem and aggravation of the brainstem edema. Besides 


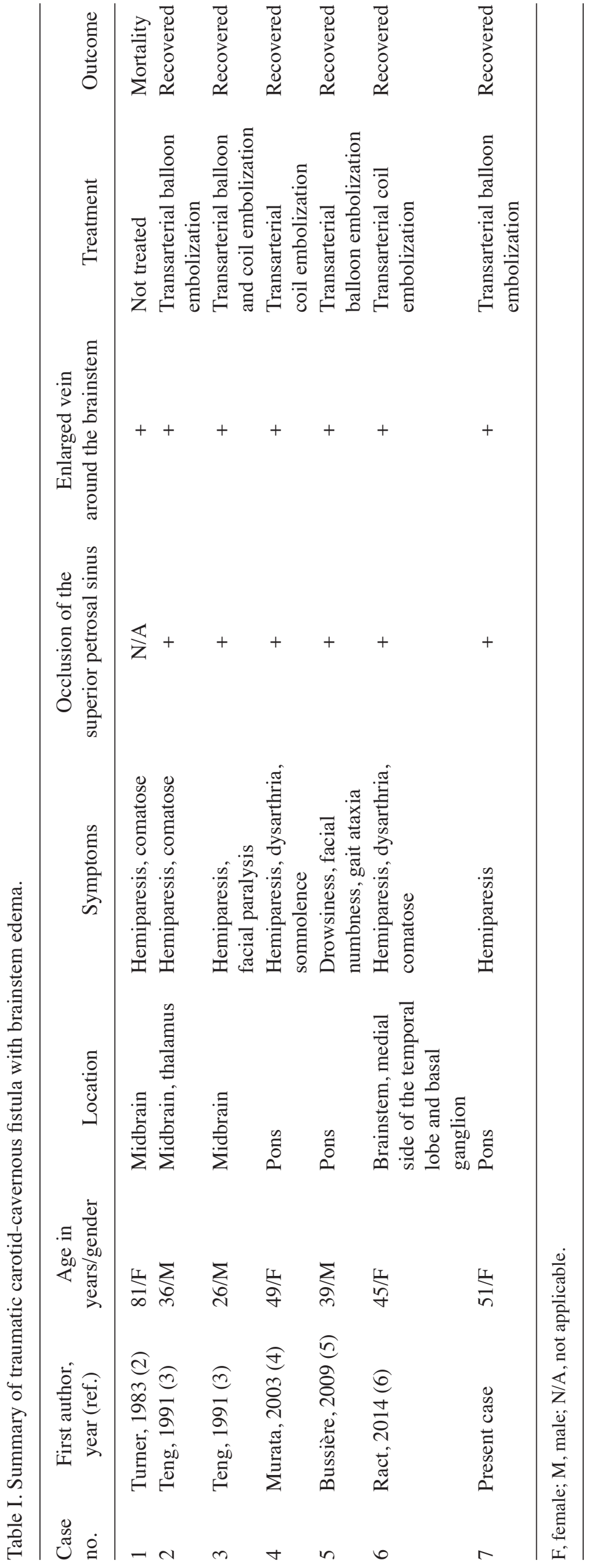


occlusion of the superior petrosal sinus and enlargement of the veins, hypoplasia of the basal vein of Rosenthal was observed in cases with CSDAVF and TCCF with brainstem edema $(6,15)$. In combination, these findings suggest that poor venous drainage may be a main cause of brainstem edema with TCCF.

The cause of superior petrosal sinus occlusion in TCCF remains unclear. Since spontaneous occlusion of a bilateral TCCF and spontaneous thrombosis of CCF following failed transarterial balloon occlusion have been reported $(17,18)$, we speculate that stasis of venous flow and loss of venous pressure in TCCF may lead to stasis of blood flow in the superior petrosal sinus. Similar conditions have been reported in carotid-cavernous dural arteriovenous fistula $(19,20)$ and in the ophthalmic vein following TCCF (21); therefore, thrombus formation due to stasis of the venous flow likely contributes to occlusion of the superior petrosal sinus in TCCF. Other factors may also contribute to occlusion of the superior petrosal sinus, however, including induction of thrombus formation by contrast agents either by directly acting on endothelial cells or via the accumulation of white and red blood cells. This may occur as a result of the accumulation of contrasts in the cavernous sinus, since contrasts do not induce thrombus formation in normal blood vessels $(22,23)$. In all six cases available with brainstem edema caused by TCCF, brainstem edema and its associated symptoms occurred following the use of contrast materials (2-6). In the present case, there was mild brainstem edema on MRI during the use of contrast materials, but the brainstem edema was aggravated following the use of contrast materials. It is therefore possible that the use of contrasts may lead to brainstem edema.

Brainstem edema caused by TCCF is associated with venous congestion, which can be effectively treated with transarterial coil or balloon embolization (Table I). Since ischemia and infarction do not occur, the prognosis of TCCF with brainstem edema is similar to that of brain edema caused by thrombosis of the intracranial venous sinus (24). Five patients who were treated with transarterial coil or balloon embolization achieved satisfactory outcomes. In the present case, the hemiparesis and brainstem edema disappeared with the transarterial balloon embolization.

In conclusion, occlusion of the superior petrosal sinus commonly occurs in TCCF, which can lead to poor venous drainage in the brainstem. Increased venous pressure in the superior petrosal sinus and clival venous plexus further blocks the venous drainage in the brainstem, leading to brainstem edema and its associated symptoms, such as hemiparesis. Use of contrasts for angiography may aggravate the brainstem edema. Relief of brainstem edema and brainstem edema-associated clinical symptoms can be achieved with transarterial coil or balloon embolization of the TCCF to reduce the drainage pressure in the brainstem veins.

\section{References}

1. Kato M, Ikegame Y, Toyoda I, Ogura S, Kitajima H, Yoshimura S and Iwama T: Hemispheric laminar necrosis as a complication of traumatic carotid-cavernous sinus fistula. Neurol Med Chir (Tokyo) 49: 26-29, 2009.

2. Turner DM, Vangilder JC, Mojtahedi S and Pierson EW: Spontaneous intracerebral hematoma in carotid-cavernous fistula. Report of three cases. J Neurosurg 59: 680-686, 1983.
3. Teng MM, Chang T, Pan DH, Chang CN, Huang CI, Guo WY, Chen CC, Pang RG and Lee LS: Brainstem edema: An unusual complication of carotid cavernous fistula. AJNR Am J Neuroradiol 12: 139-142, 1991.

4. Murata H,Kubota T, Murai M, Kanno H, Fujii S and Yamamoto I: Brainstem congestion caused by direct carotid-cavernous fistula - case report. Neurol Med Chir (Tokyo) 43: 255-258, 2003.

5. Bussière M, Lownie SP, Pelz DM and Nicolle D: Direct carotid-cavernous fistula causing brainstem venous congestion. J Neuroophthalmol 29: 21-25, 2009.

6. Ract I, Drier A, Leclercq D, Sourour N, Gabrieli J, Yger M, Nouet A, Dormont D, Chiras J and Clarençon F: Extensive basal ganglia edema caused by a traumatic carotid-cavernous fistula: A rare presentation related to a basal vein of Rosenthal anatomical variation. J Neurosurg 121: 63-66, 2014.

7. de Keizer R: Carotid-cavernous and orbital arteriovenous fistulas: Ocular features, diagnostic and hemodynamic considerations in relation to visual impairment and morbidity. Orbit 22: 121-142, 2003.

8. Rhoton AL Jr: The cavernous sinus, the cavernous venous plexus and the carotid collar. Neurosurgery 51 (4 Suppl): S375-S410, 2002.

9. Fusco MR and Harrigan MR: Cerebrovascular dissections: A review. Part II: Blunt cerebrovascular injury. Neurosurgery 68 : 517-530, 2011.

10. Chang CM and Cheng CS: Late intracranial haemorrhage and subsequent carotid-cavernous sinus fistula after fracture of the facial bones. Br J Oral Maxillofac Surg 51: e296-e298, 2013.

11. Asano T, Houkin K, Moriwaki T, Niiya Y and Mabuchi S: Case of direct carotid-cavernous fistula presenting with subarachnoid hemorrhage. No Shinkei Geka 40: 235-239, 2012 (In Japanese).

12. Ringer AJ, Salud L and Tomsick TA: Carotid cavernous fistulas: Anatomy, classification and treatment. Neurosurg Clin N Am 16: 279-295, 2005.

13. Kalra VB, Malhotra A and Matouk CC: Teaching neuroimages: Susceptibility-weighted MRI: First clue to DAVF complicating sinovenous thrombosis. Neurology 80: e228, 2013.

14. Blanc R, Maia Barros AD, Brugieres P, Meder JF and Gaston A: Cavernous sinus dural arteriovenous fistula complicated by edematous cerebral lesions from venous etiology. J Neuroradiol 31: 220-224, 2004 (In French).

15. Miyamoto N, Naito I, Takatama S, Shimizu T, Iwai T and Shimaguchi H: Clinical and angiographic characteristics of cavernous sinus dural arteriovenous fistulas manifesting as venous infarction and/or intracranial hemorrhage. Neuroradiology 51: 53-60, 2009.

16. Curé JK, Van Tassel P and Smith MT: Normal and variant anatomy of the dural venous sinuses. Semin Ultrasound CT MR 15: 499-519, 1994.

17. Churojana A, Chawalaparit O, Chiewwit P and Suthipongchai S: Spontaneous occlusion of a bilateral post traumatic carotid cavernous fistula. Interv Neuroradiol 7: 245-252, 2001.

18. Uchino A, Takase Y, Koizumi T and Kudo S: Spontaneous thrombosis of a high-flow carotid-cavernous fistula after failed transarterial balloon occlusion. Interv Neuroradiol 10: 253-256, 2004.

19. Sasaki H, Nukui H, Kaneko M, Mitsuka S, Hosaka T, Kakizawa T, Kimura R, Nagaseki Y and Naganuma H: Long-term observations in cases with spontaneous carotid-cavernous fistulas. Acta Neurochir (Wien) 90: 117-120, 1988.

20. Komiyama M, Nakajima H, Nishikawa M and Kan M: Traumatic carotid cavernous sinus fistula: Serial angiographic studies from the day of trauma. AJNR Am J Neuroradiol 19: 1641-1644, 1998.

21. Iseki S, Ito Y, Nakao Y, Yamamoto T and Mori K: Proptosis caused by partially thrombosed orbital varix of the superior orbital vein associated with traumatic carotid-cavernous sinus fistula - case report. Neurol Med Chir (Tokyo) 50: 33-36, 2010.

22. Nishijima M, Iwai R, Horie Y, Oka N and Takaku A: Spontaneous occlusion of traumatic carotid cavernous fistula after orbital venography. Surg Neurol 23: 489-492, 1985.

23. Castillo M, Silverstein M, Hoffman JC Jr and Barrow D: Spontaneous thrombosis of a direct carotid cavernous sinus fistula: Confirmation by Gd-DTPA-enhanced MR. AJNR Am J Neuroradiol 10 (5 Suppl): S75-S76, 1989.

24. Gupta RK, Bapuraj JR and Khandelwal N and Khurana D: Prognostic indices for cerebral venous thrombosis on CT perfusion: A prospective study. Eur J Radiol 83: 185-190, 2014. 